\title{
5-Carboxylate Substituted Piperazine-2-ones as Potent and Selective MMP-2 Inhibitors
}

\author{
Yong Jun Chung, Keyong-Ho Lee, Sang-Hoo Park, Chang-Woo Cho, and Min Su Han ${ }^{\ddagger}$ \\ Kolon Life Science Research Institute, Iongin, Gueonggi 449-797, Korea. ${ }^{*}$ E-mail ; ,chungakolon.com \\ 'Department of Chemistry Kinngpook National Chiversitw, Daegu 702-701, Korea \\ -Department of Chemistr, Chung-1ng Lniversity, Seoul 156-756, Korea \\ Recerved 1Farch 12,2008
}

Key Words : MMP inhibitors. Piperazinones. Peptidomimetics

Matrix metalloproteinases (MMP) are metalloproteases. which contain zinc at their activation site. ${ }^{3}$ These enzymes decompose and reconstruct structural proteins in the extracellular matrix such as membrane collagen, aggrecan. fibronectin, and laminin. As a result, MMPs play an important role in the development of chronic diseases such as multiple sclerosis, arthritis. fibrosis and other inflanumation, and the growth and metastasis of malignant tumors ${ }^{2,3}$ For this reasons, MMPs are attractive targets for drug candidates which can inhibit and potentially treat such diseases. ${ }^{+}$

The majority of MMPs are divided into four groups that include collagenase (MMP-1, -8, -13). gelatinase (MMP-2. -9). stromelysins (MMP-1. - 10, -3) and membrane type MMPs (MMP-14. -15, 16, 17).$^{3}$ Of these groups, the gelatinase MMPs are an attractive target because the inhibitors of MMP-2 are useful for anti-cancer therapy. Many MMP-2 inhibitors were developed and underwent clinical tests. However, owing to the inhibitors bearing low selectivity to MMP-2. the majority of these compounds were unsuccessful in the clinical tests. ${ }^{6}$ We. therefore, have concentrated our efforts on the development of selective MMP-2 inhibitors while sparing other MMPs. Herein we report the use of piperazinones bearing zinc binding groups (ZBG) for selective inhibition of MMP-2.

MMPs inhibitors based upon piperazinone have some advantages over substrate analog inhibitors. Substrate ana$\log$ inhibitors suffer from hydrolysis by peptidase in wivo. whereas conformationally restricted peptidomimetics such as piperazinone are not hydrolyzed by peptidase and retain their activity in wivo. ${ }^{7}$ Moreover. piperazinone based inhibitors can be easily modified with diverse $P_{1}$ and $P_{2}$ substituents that can improve inhibitory potency and selectivity

\section{(Figure 1). ${ }^{8}$}

Firstly, we synthesized piperazinone derivatives containing a lydroxamic acid as the zinc binding group because the hydroxamic acid has the high affinity for the catalytic zinc ion in the active site of MMPs and can bind to Glu-202 and Ala-165 in active site of MMPs via lydrogen bond. ${ }^{9}$

The synthetic route for creating piperazinone derivatives containing a hydroxamic acid group was outlined in Scheme 1. ${ }^{101}$

Piperazinone derivatives were evaluated for inhibition of MMP-2, MMP-9 and MMP-1, Table $1 .{ }^{11}$ Although these inhibitors strongly inhibited MMP-2. they suffered from just 10-fold selectivity over MMP-1. To improve selective inhibition of the MMP-2 over other MMPs, we examined piperazinone derivatives containing a carboxylic acid group as a selective MMP-2 inhibitor. By replacing the strong ZBG hydroxamic acid with the relatively less stronger $Z B G$ carboxylic acid. we expected that the selectivity of the

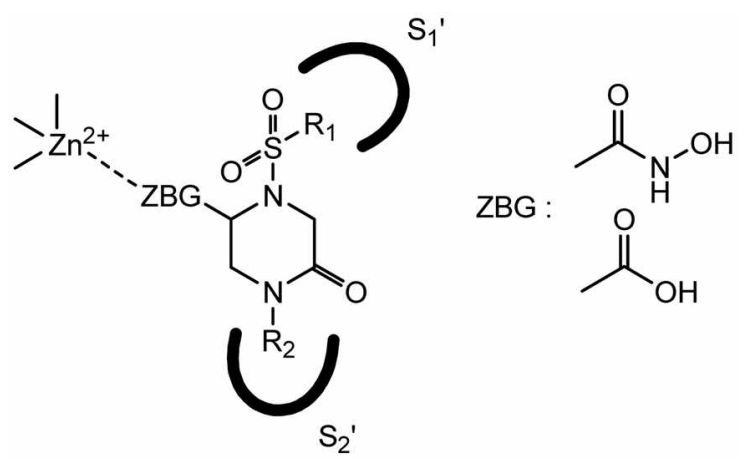

Figure 1. Schematic representation for binding mode of piperazinone based MMP-2 inhibitors.

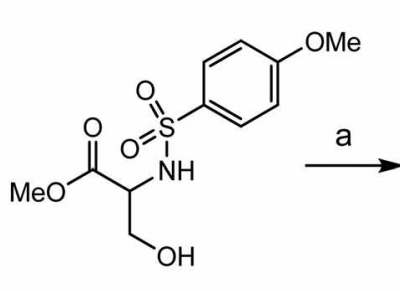

1

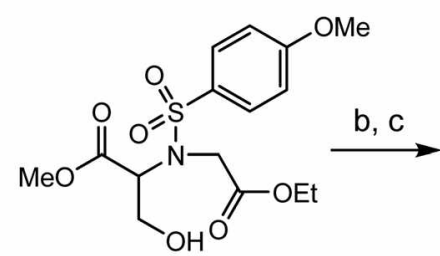

2<smiles>[R]N1CC(C(=O)NO)N(S(=O)(=O)c2ccc(OC)cc2)CC1C(=O)NO</smiles>

3

Scheme 1. Reagents and conditions: (a) Ethyl bromoacetate, $\mathrm{K}_{2} \mathrm{CO}_{3}, \mathrm{DMF} ;$ (b) $\mathrm{MsCl}, \mathrm{Et}_{3} \mathrm{~N}_{2} \mathrm{CH}_{2} \mathrm{Cl}_{2} ; \mathrm{R}-\mathrm{NH}_{2}, \mathrm{CH}_{2} \mathrm{Cl}_{2}$; (c) $\mathrm{LlOH}_{1} \mathrm{OHF}$; (d) O-Benzyl-hydroxylamine, DCC: (e) $\mathrm{Pd} / \mathrm{C}, \mathrm{H}_{2}, \mathrm{EtOH}$ 
Table 1. $\mathrm{IC}_{50}$ values for hydroxannic acid based inhibitors against MMP-1, MMP-2, and MMP-9<smiles>COc1ccc(S(=O)(=O)N2CC(=O)N(P)CC2C(=O)NO)cc1</smiles>

\begin{tabular}{|c|c|c|c|c|}
\hline \multirow{2}{*}{ Compds } & \multirow{2}{*}{$\mathrm{R}$} & \multicolumn{3}{|c|}{$\mathrm{IC}_{50}(\mu \mathrm{M})$} \\
\hline & & MMP-1 & MMP-2 & MMP-9 \\
\hline $4 a$ & $\mathrm{H}$ & 0.089 & 0.051 & 0.036 \\
\hline $4 b$ & $\mathrm{CH}_{3}$ & 0.351 & 0.014 & 0.013 \\
\hline $4 c$ & $\mathrm{CH}_{3}\left(\mathrm{CH}_{2}\right)=$ & 0.485 & 0.040 & 0.052 \\
\hline $4 d$ & Allyl & 0.292 & 0.016 & 0.009 \\
\hline te & $\mathrm{Ph}-\mathrm{C} \mathrm{H}_{2}$ & 0.013 & 0.005 & 0.015 \\
\hline $4 f$ & $o$-Pyridine- $\mathrm{CH}_{2} \cdot \mathrm{HCl}$ & 0.461 & 0.170 & 0146 \\
\hline $4 g$ & $o$-Pytridine- $\mathrm{C}_{2} \mathrm{H}_{+} \cdot \mathrm{HCl}$ & 0.353 & 0.084 & 0.084 \\
\hline th & Piperidine- $\mathrm{C}_{2} \mathrm{H}_{4} \cdot \mathrm{HCl}$ & 0.176 & 0.030 & 0.027 \\
\hline $4 \mathbf{i}$ & Morphorine- $\mathrm{C}_{2} \mathrm{H}_{4} \cdot \mathrm{HCl}$ & 0.200 & 0.038 & 0.114 \\
\hline
\end{tabular}

inhibitors against MMPs is strongly dependent on the substituents on the piperazinone derivatives. Fron X-ray crystallographic analysis. nuclear magnetic resonance (NMR) analysis and homology modeling. the $S_{1}$ ' pockets of MMP-2 are a relatively deeper than these of MMP-I and, consequently. incorporation of an extended $\mathrm{P}_{l}^{\prime}$ group (e.g. bromo-biphenyl) leads to selective inhibition. $1 \hat{2}, 13$

Carboxylic acid based inhibitors with bromo-biphenyl moiety were synthesized based upon Scheme I and evaluated for the inlibition of MMPs including MMP-1, MMP-2. MMP-9, MMP-13, and MMP-14, Table 2. As expected. compound 7 a containing the bromo-biphenyl group as the $\mathrm{P}^{\prime}$ group was found to have the high potency $\left(\mathrm{IC}_{50}=0.7\right.$ nM) for MMP-2 with the high selectivity over MMP-l and MMP-14. However, compound 7a exhibited low selectivity over MMP-13 which also has a relatively deep hydrophobic $S_{1}$ ' pocket. We explored the structure activity relationship (SAR) of compound $7 \mathrm{a}$ with the intent of improving its selectivity while maintaining the inhibitory potency by modifying the $\mathrm{P}_{2}^{\prime}$ substituent with versatile alkyl chains.

Modification of the $\mathrm{P}_{2}{ }^{\prime}$ substituent with different alkyl chains induced the strong effect on the inhibitory potency and selectivity. Especially. compound $7 \mathrm{~b}$ was an active inhibitor $\left(\mathrm{IC}_{\text {Si }}=1 \mathrm{nM}\right)$ of MMP-2 with > 1000-fold selectivity over MMP-1, MMP-13. and MMP-14 by SAR. Moreover. compound $7 \mathrm{~h}$ had the high inhibitory potency against MMP2 with greater selectivity than the hydroxamic acid based inhibitor (Marimastat) and the carboxylic acid based inhibitor (BAY 12-9566). ${ }^{+14}$

In conclusion, carboxylic acid derivatives of piperazin-2one were synthesized and evaluated as the inhibitors of MMP-2. The derivatives were highly potent inhibitors of MMP-2 with > 1000 fold selectivity over MMP-1. MMP-13. MMP-14 and may thus be potentially useful scaffold in the
Table 2. IC 50 values for carboxylic acid based inhibitors against MMP-1, MMP-2, MMP-9, MMP-13 and MMP-14

\begin{tabular}{|c|c|c|c|c|c|}
\hline & & $\begin{array}{l}\text { 7a R }=\mathrm{CH}_{3} \\
\text { 7b R }=\mathrm{CH}_{3} \\
7 \mathrm{c} \mathrm{R}=\mathrm{CH}_{3} \\
\text { 7d R }=\mathrm{CH}_{3}\end{array}$ & $\begin{array}{l}\left.\mathrm{CH}_{3}\right)_{3} \\
\left.\mathrm{CH}_{2}\right)_{5} \\
\left.\mathrm{CH}_{2}\right)_{3}\end{array}$ & $\begin{array}{l}\text { 7e R }=\mathrm{CH}_{3} \\
7 f \quad R=\mathrm{CH}_{3} \\
7 \mathrm{~g} \mathrm{R}=\mathrm{CH}_{3} \\
7 \mathrm{~h} \mathrm{R}=\mathrm{CH}_{3}\end{array}$ & $\begin{array}{l}\left(\mathrm{CH}_{2}\right)_{9} \\
\left(\mathrm{CH}_{2}\right)_{11} \\
\left(\mathrm{CH}_{2}\right)_{13} \\
\left(\mathrm{CH}_{2}\right)_{15}\end{array}$ \\
\hline \multirow{2}{*}{ Compds } & \multicolumn{5}{|c|}{ IC $s_{1}(\mu \mathrm{M})$} \\
\hline & MMP-I & MMP-2 & MMP-9 & MMP-13 & MMP-14 \\
\hline $7 a$ & 3.857 & 0.0007 & 0.0016 & 0.008 & $>10$ \\
\hline $7 b$ & $>25$ & 0.001 & 0.152 & 1.130 & $>10$ \\
\hline $7 c$ & $>25$ & 0.001 & 0.076 & 0.0082 & $>10$ \\
\hline $7 d$ & $>25$ & 0.004 & 0.089 & 0.917 & $>10$ \\
\hline $7 e$ & $>25$ & 0.074 & 2.235 & 2.460 & $>10$ \\
\hline $7 f$ & $>25$ & 1.195 & 15.12 & 2.350 & $>10$ \\
\hline $7 \mathrm{~g}$ & $>25$ & 1.821 & $>25$ & $>10$ & $\mathrm{nd}^{a}$ \\
\hline $7 \mathrm{~h}$ & $>25$ & 0.981 & $>25$ & $>10$ & $\mathrm{nd}^{a}$ \\
\hline Marimastat & $0.0008^{b}$ & 0.0004 & 0.0008 & $0.0012^{\circ}$ & $n d^{\prime \prime}$ \\
\hline BAY 12-9566 & $>5^{-t}$ & $0.011^{\circ}$ & $0.301^{3}$ & $1.47^{\circ}$ & $\mathrm{nd}^{\prime \prime}$ \\
\hline
\end{tabular}

${ }^{a} \mathrm{IC}_{50}$ was not detemined. ${ }^{b} \mathrm{IC}_{5 \mathrm{U}}$ Literature values (ref. 5.14 )

design of inluibitors that are effective for other zinc proteases of medicinal interest.

\section{References}

1. Beckett, P. P.; Davidson, A. H.: Drummond, A. H.: Huxley. P. Whittaler. M. Dnug Disc. Today 1996. 1. 16-26.

2. Egeblad, M.: Werb, Z. Natw? Rev Cancer 2002, 2. 161-174.

3. Hu. I.: Van den Steen. P. E.: Sang. S.-Q. A.: Opdenakiker. G. Nature Rev: Drng Dis. 2006. 6. 480-498.

4. Whittaker. M.: Hloyd. C. D.: Brown. P.: Gearing. A. J. H. Chent Rev 1999, 99. $2735-2776$.

5. Johnson, L. L.: Dyer, R: Hupe. D. J. Cum: Opin. Cham. Biol. 1998. $2,466-471$.

6. Drummond. A. H.: Beckett. P.: Browi1. P. D.: Bone. E. A.: Davidson. A. H.: Galloway. W. A.: Gearing. A. T. H.: Huxley. P.: Laber. D.: McCoutt. M.: Whittaker. M.: Wood. L. M.: Wright. A. Am. X. Y. Acad Sci. 1999. 878, 228-235.

7. Patani, G. A.; LaVoie, E. J. Chem. Rev 1996, 96, 3147-3176.

8. Pikul, S.: Dunham, K. M.; Almstead. N. G.: De, B.: Natchus. M. G.: laiwo. Y. (O.: Willianns. L. E.: Hynd. B. A.: Hsieh. L. C.: Januse. M. J.: Gu. H.: Mieling. G. E. Bioong Med. Chem. Lett. 2001. 11. 1009-1013.

9. Babine, E. R.; Bender. S. L. Chem. Rev: 1997, 97, 1359-1472.

10. Chung. Y. J.: Kim. Y.C.: Park. H.-J. Bull. Kor: Chem. Soc. 2002. 23. 1481-1482.

11. Weingarten. H.: Feder. T. Anal. Biochem. 1985. 147. 437-440.

12. Lovejoy. B.: Cleasby. A.: Hassell. A. M.: Longley. K.: Luther. M. A.: Weigl. D.: McGeehat1. G: McElroy. A. B.: Drewry. D.: Lanbert, M. H.: Jordan. S. R. Science 1994. 263. 375-377.

13. Gooley, P. R.: O'Connell. J. F.: Marcy, A. I.: Cuca, G. C.: Salowe. S. P.: Bush, B. L.; Hermes. J. D.: Esser, C. K: Hagmann. W. K: Springer. T. P. Johnson. B. A. Struct. Biol 1994. 1. 111-118.

14. Wada. C. K.: Holms. T. H.: Curtin. M. L.: Dai. Y.: Florjancic. A S.: Garland. R. B.: Guo. Y:: Heyman. H. R.: Stacey. J. R.: Steinman. D. H.: Albert, D. H.; Bouska. J. J.; Elmore, I. N.: Goodtellow, C. L.; Marcotte. P. A.: Tapang, P.: Morgan. D. W: Michaelides. M. R.; Davidsen, S. K. J. Hed Chem 2002. 45.219-232. 